these conditions, so that all the label can be assumed to represent phenylalanine-sRNA. This result is consistent with the recent findings of Cannon, Krug and Gilbert', but the experiment differs from theirs in that it tested for the binding of a specific $s \mathrm{RNA}$ and in the presence, rather than in the absence, of messenger.

Details of these experiments, as well as experiments demonstrating that chloramphenicol does not interfere with the formation of peptide linkages per se, will be reported in a forthcoming communication.

This work was supported by U.S. National Science Foundation grant $G B-578$ and U.S. Public Health Service grant $G M-09591$.

O. JARDETZKY

G. R. Jultan

Department of Pharmacology,

Harvard Medical School, Boston, Mass.

${ }^{1}$ Jardetzky, O., Proc. Fifth Intern. Biochem., Moscow, 157 (1961).

'Jardetzky, O., J. Biol. Chem., 238, 248 (1963).

Hahn, F. E., and Wolfe, A. D., Biochem. Biophys. Res. Comm., 6, 464 (1962).

${ }^{4}$ Rendi, R., and Ochoa, S., J. Biol. Chem., 237, 3711 (1962).

Weisberger, A. S., Armentrout, S., and Wolfe, S., Proc. U.S. Nat. Acad. Sci., 50, 86 (1963).

'Matthaei, J. H., and Nirenberg, M., Proc. U.S. Nat. Acad. Sci., 47, 1580 (1961).

'Bock, R. M. (personal communication).

"Gierer, A., and Sehramm. G., Nature, 177, 702 (1956).

${ }^{9}$ Cannon, M., Krug, R., and Gillbert, W., J. Mol. Biol., 7, 360 (1963).

\section{Hydroxylation of Phenoxyacetic Acid and Anisole by Aspergillus niger (van Tiegh)}

Investigations of the hydroxylating activity of $A$. niger have been carried out mainly in connexion with tho metabolism of the phenoxy- and naphthyloxy-alkylcarboxylic acids ${ }^{1,2}$, at least in part because of the importance of these compounds as herbicides. We have been examining the action of this organism, using van Tiegh strain, on various benzenoid compounds, and since our results for the hydroxylation of phenoxyacetic acid differ fundamentally from those obtained with van Tiegh (Mulder) strain ${ }^{1}$, we wish to report our findings for both this compound and the related aromatic ether, anisole.

A replacement culture technique was used with both substrates. The organism was grown on a glucose salt medium containing 1 per cent malt at $25^{\circ}$ for $72 \mathrm{~h}$. The medium was then removed and the mycelium was washed and, in the case of phenoxyacetic acid, resuspended in a $0.001 \mathrm{M}$ solution of the substrate at $p \mathrm{H} 8.0(0.01 \mathrm{M}$ phosphate buffer). After incubation for periods of 3,5 and 7 days, carried out on a rotary shaker at $25^{\circ}$, the medium was analysed for phenolic products. Because of anisole's insolubility and toxicity, the technique was slightly modified in this case: the substrate was dissolved in about seven times its volume of carbon tetrachloride and this solution was placed at the bottom of each culture flask.

The main product from phenoxyacetic acid was identical to 2-hydroxyphenoxyacetic acid in the following respects: (a) $R_{F} 0.44$ at room temperature, in butanolethanol-3 M ammonia (4:1:5) ; (b) colour reactions with the detecting reagents ferric chloride-potassium ferricyanide (pink) and diazotized $p$-nitroaniline (yellow); (c) ultra-violet absorption ( $\lambda_{\max } 275 \mathrm{~m} \mu$ at $p \mathrm{H} 2.0$ and $288 \mathrm{~m} \mu$ at $p \mathrm{H} 11 \cdot 0)$; $(d)$ retention time in gas chromatographic analysis on diethyleneglycol adipate polyester. Authentic 4-hydroxyphenoxyacetic acid had $R_{F}=0 \cdot 27$, gavo blue and red spots, respectively, with the two detecting reagents, and had $\lambda_{\max } 287 \mathrm{~m} \mu$ at $p H \quad 2.0$ and $306 \mathrm{~m} \mu$ at $p H 11 \cdot 0$, and material with these properties could not be detected in the oxtract. Gas chromatographic analysis revealed the presence of phenol as a minor product.

The product from anisole was found by paper chromatography to contain guaiacol (o-methoxyphenol), but the meta- and para-isomers were not detected. Gas chromatography on diethyleneglycol adipate polyestor, using conditions in which phenol and the three methoxyphenols were completely resolved, showed that at least 95 per cent of the mono-hydroxylated product was the ortho-derivative, but the occurrence of very small peaks with retention times equal to those of the meta- and para-derivatives indicated that the possibility of their being present could not be entirely excluded. Gas chromatography also showed that phenol was presont in almost as large a quantity as guaiacol when incubation was stopped after 3 days but in lower yield relative to guaiacol after 7 days. Reduction of the $p \mathrm{H}$ of the incubation medium from 8 to 7 lowered the yield of hydroxylated products, while at $p H 6$ no hydroxylation could be detected.

The reactions of these aromatic ethers indicate that an ortho-hydroxylase is produced by the organism. This is in contrast to the report that the van Tiegh (Mulder) strain is relatively non-specific in its hydroxylating activity towards phenoxyacetic acid, giving both ortho- and parahydroxy derivatives with predominance of the latter ${ }^{1}$. It appears that the difference arises from a difference in the strains used, and a further study of this, as well as of the enzymatic nature of the hydroxylating system, is in progress. Finally, it is interesting to note that $O$-de-alkylation occurs with both substrates, as it does also in the liver microsome hydroxylating system ${ }^{3}$.

Sheil. M. Bocks

J. R. Lindsay Smith

R. O. C. NORMAN

Dyson Perrins Laboratory,

South Parks Road, Oxford.

1 Woodcock, $\mathrm{D}_{.}$, in Phenolics in Plants in Health and Disease, edit. by Pridham, $\vec{J}$. B. (Pergamon Press, 1960).

${ }^{2}$ Faulkner, J. K., and Woodeock, D., J. Chem. Soc., 5397 (1961).

${ }^{3}$ Brodie, B. B., et al., Science, 121, 603 (1955). Axelrod, J., J. Pharmacul. Exp. Therap., 115, 259 (1955).

\section{PHYSIOLOGY}

\section{Inhibition and Reversal of Swelling of Rat Heart Sarcosomes by Reduced Diphosphopyridine Nucleotide}

ADENosine triphosphate (ATP) has been known for some time to inhibit or reverse the swelling of isolated rat liver mitochondria ${ }^{1-5}$. Contraction by A'TP of sarcosomes from pigeon-breast muscle ${ }^{6}$ and from rat heart 7 has also been reported. The results hitherto available suggested that the contraction of mitochondrial particles is unique to ATP, since triphosphates of other nucleotides were found to be ineffective ${ }^{4}$. As an outcome of investigations on the swelling-contraction characteristics of rat heart sarcosomes it has now been found that while diphosphopyridine nucleotide (DPN) is a powerful inducer of partieulate swelling ${ }^{8,9}$, the reduced nucleotide (DPNH) is as effective as ATP to contract swollen sarcosomos.

Male Sprague-Dawley rats (300-370 g) fed 'Purina Chow' were used. Isolation of the heart sarcosomes ${ }^{8}$ and the swelling tests (by the photometric method) were patterned on procedures $8,10,11$ previously described. The test system used here is given in the legend to Fig. 1. All experiments were carried out with three to five animals. Examination by phase-contrast microscopy $(\times 970)$ under blue-green light was used to ascertain the morphological integrity and mobility of the isolated particles, and, furthermore, that the increase of optical density measured photometrically following addition of DPNH or ATP corresponded to actual contraction of the sarcosomes.

Investigations of the dependence on $p \mathbf{H}$ of swelling carried out previously with various inducers and using liver mitochondria ${ }^{1,3,10}$, and at present using heart sarcosomes in hypotonic medium, indicatc that for swelling the optimum $p \mathrm{H}$ is $7 \cdot 4-7 \cdot 5$. On the other hand, the $p \mathrm{H}$ range for reversal or inhibition of sarcosomal swelling is different. There is no reversal at $p \mathbf{H} 6 \cdot 8-7 \cdot 2$ of hypotonic (Fig. I) or thyroxine-induced (Fig. 2) swelling by addition of DPNH up to a final concentration of $1.5 \times 10^{-2} \mathrm{M}$. 